\title{
Study of constrained Brownian motion of nanoparticles near an interface using optical tweezers
}

\author{
Hui Yang*, Matteo Cornaglia, Raphäl Trouillon, Thomas Lehnert, Martin A. M. Gijs \\ Laboratory of Microsystems, École Polytechnique Fédérale de Lausanne, Lausanne, Switzerland \\ $\mathrm{CH}-1015$
}

\begin{abstract}
We demonstrate a method to determine the Brownian motion and the diffusion coefficient of a nanoparticle in water in a plane that is parallel to a solid boundary and as function of the distance normal to that boundary by using an optical tweezers instrument. A solution of $190 \mathrm{~nm}$-diameter fluorescent polystyrene nanoparticles in de-ionized (DI) water is introduced in a micro-chamber built from two thin glass substrates. A single particle is trapped by the tweezers and optically moved in the $z$-direction normal to a substrate. By analyzing a scatter plot of the time-dependent positions of the nanoparticle in the $x-y$ plane in a histogram, the diffusion coefficient parallel to the substrate of the Brownian particle constrained by the substrate is determined as a function of the distance between the substrate and the nanoparticle. The experimental results indicate the increased drag effect on the nanoparticle when it is close to the substrate, as evidenced by an experimental diffusion coefficient nearby the substrate that is about half of that of the particle in the bulk fluid.
\end{abstract}

Keywords: Brownian motion, nanoparticle, optical tweezers

\section{INTRODUCTION}

Optical tweezers can be used for non-intrusive manipulation of micro/nanometer-sized objects by focused laser light $[1,2]$. In this technique, laser light is focused in a spot with a dimension corresponding to the diffraction limit of a high numerical aperture (NA) microscope objective to produce a single-beam gradient-force optical trap. A transparent object with refractive index larger than that of its surrounding medium is pulled toward the center of the trap by the forces that arise when the light is refracted by the object. Optical tweezers have been widely used in many contexts in biological physics [3,4], for example, in single molecule studies of molecular motors $[5,6]$, other proteins and polymers $[7,8]$, and in surgery at the cellular level $[9,10]$. In some of these contexts, the tweezers are only used to grab and hold the sample. In other contexts, they are used to exert a prescribed force or to measure forces [11-13]. If a particle held by the optical tweezers is displaced from the focal region of the trap by an external force, the displacement is limited by the optical restoring force for displacements smaller than half the radius of the particle within the focal region. The amount of displacement is thus a direct measure of the external force applied [14]. In order to determine the force to which a particle is exposed, the trajectory of a particle in an optical trap is therefore tracked by an optical position sensor such as a quadrant photodiode (QPD). A particle trapped in optical tweezers is unavoidably exposed to Brownian forces that induce a random walk around its equilibrium position. The extent of fluctuation in the position of the particle is conveniently monitored by the QPD. Thus, the tweezers are also widely used to determine the Brownian motion of a trapped particle and to measure the local viscosity $[15,16]$.

In the bulk of the fluid, far away from the boundary, the diffusivity of an isolated particle follows the Stokes-Einstein relation, which balances the fluid thermal energy with the hydrodynamic mobility of the particle. In the presence of a nearby solid boundary, however, a particle experiences an increased drag which hinders its mobility [17,18]. The increase of the drag force is attributed to the alteration of the hydrodynamic interaction between the particle and the fluid generated by the boundary condition imposed by the nearby substrate [19]. In the applications where micro/nanometersized particles are used in close proximity to solid surfaces, understanding the hindered Brownian motions is therefore important. In this paper, we propose the measurement of the component of the Brownian diffusion parallel to a boundary by using optical tweezers instrumentation.

*hui.yang@epfl.ch; phone 4121693 6815; fax 41216935950 


\section{MATERIALS AND METHODS}

\subsection{Measurement setup}

The optical trapping and simultaneous measurement of the Brownian motion of the nanoparticles are carried out on a commercial optical tweezers instrument (NanoTracker ${ }^{\mathrm{TM}} 2$, JPK Instruments, Germany), which is constructed on a Zeiss Axio observer inverted microscope. Figure 1 shows a schematic diagram depicting the optical components used in the setup. Briefly, the trapping diode laser at $1064 \mathrm{~nm}$ is focused to a diffraction-limited spot using a $63 \times$ water-immersion microscope objective (C-Apochromat, Zeiss, Germany) with NA of 1.2. The laser beam is steered by a piezoelectric transducer-controlled mirror and, by adjusting the latter, the position of the optical trap can be varied. The light emitted from a trapped particle is detected by a QPD. A laser power of $200 \mathrm{~mW}$ at the focus is used throughout the experiments. A motorized translation stage is used to move the sample. A light-emitting diode (LED) illumination with an integrated condenser is used to align the optical path. The excitation/emission of the fluorescent particles is achieved by using a mercury arc lamp and using an appropriate fluorescent filter set and a video camera is used for observation.

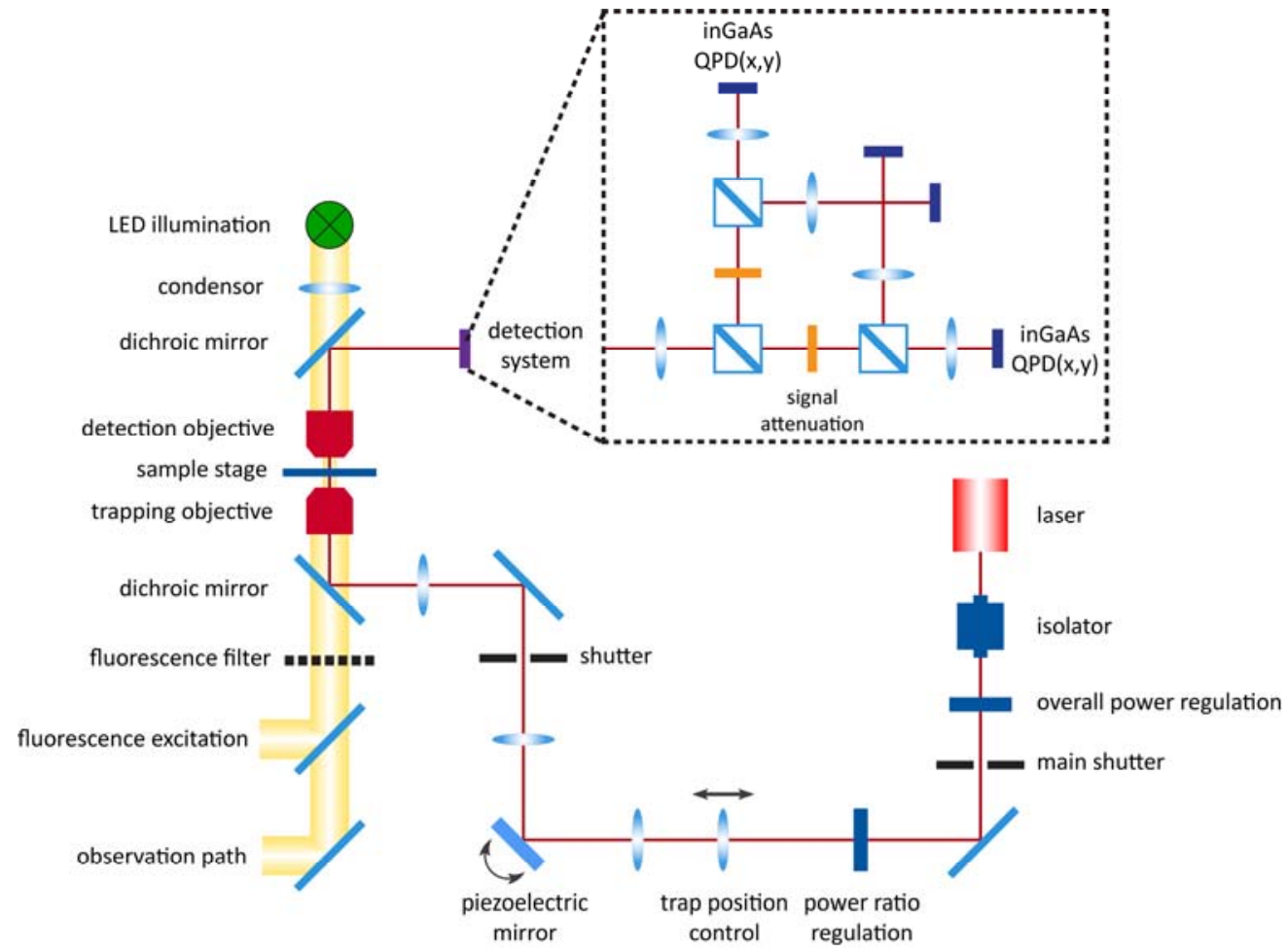

Figure 1. Experimental setup showing beam paths and crucial components of the instrument. For optical trapping, a laser beam with wavelength of $1064 \mathrm{~nm}$ is steered using a piezoelectric mirror which controls the position of the optical trap. The laser is sent to the trapping objective and used to trap an isolated nanoparticle immersed in the sample medium. After passing through the specimen, the laser light is collected by the detection objective and passed to a QPD. The latter is highly sensitive to the nanoparticle displacement as measured using back-focal-plane interferometry. LED illumination with an integrated condenser is used to align the optical path. The sample is mounted on a three-axis translation stage. For fluorescence experiments, a mercury arc lamp is used as the fluorescence excitation source and an appropriate filter set is used for detecting the fluorescence of the nanoparticles. The motion of the nanoparticle is observed by a video camera through the observation path of the setup. 


\subsection{Sample preparation}

Fluorescent nanoparticles $(190 \mathrm{~nm}$ in diameter, maximal excitation/emission wavelength: 505/515 nm, Molecular Probes, USA) are used as samples in this study. The stock solution of the fluorescent nanoparticles is mixed by a rotary mixer before being diluted with DI water in successive steps into a final concentration of $1.2 \times 10^{4}$ beads per milliliter. Two kinds of cover glasses $(22 \mathrm{~mm} \times 22 \mathrm{~mm}$ and $24 \mathrm{~mm} \times 60 \mathrm{~mm}, 0.15 \mathrm{~mm}$ in thickness, Carl Roth GmbH, Germany) are carefully sonicated with cleansing solution ( $95 \%$ ethanol and $5 \%$ methanol), rinsed with DI water, and then dried by compressed air. The two glass cover slides are used to form a square or rectangular micro-chamber that allows easy spread of the nanoparticle solution. The micro-chamber has an inner height of $\sim 100 \mu \mathrm{m}$ by using as spacer thermal glue that is uniformly dispersed on the two opposite edges of the small glass cover slide. Before measurement, a droplet of bead solution with $50 \mu \mathrm{L}$ in volume is introduced into the micro-chamber and the latter is sealed with nail polish to avoid evaporation.

\section{RESULTS AND DISCUSSION}

A hard particle with radius $a$, moving with velocity $\mathbf{V}$ in an unbounded quiescent fluid with viscosity $\eta$, experiences a hydrodynamic drag force opposite to its direction of motion. If there is no slip at the boundary between the particle and the fluid, in the low Reynolds number condition, the drag force is (Stokes law)

$$
\mathbf{F}_{\text {drag }}=-6 \pi \eta a \mathbf{V}
$$

The diffusivity $D_{0}$ of the particle can be calculate solely from the balance of the thermal kinetic motion with the viscous drag force

$$
D_{0}=\frac{k_{B} T}{6 \pi \eta a}
$$

where $k_{B}$ is the Boltzmann constant and $T$ is the fluid temperature in the absolute Kelvin scale.

When the particle is in the vicinity of a solid boundary (as illustrated in Figure 2), the Brownian motion can be substantially hindered. As the distance between the particle and the boundary decreases, the drag force acting on the particle increases with respect to Eq. (1). The increased Stokes drag force is attributed to the hydrodynamic interaction between the particle and the boundary. Because of the linearity of the Stokes equations, the drag force can be separated into independent components for particle motion parallel and perpendicular to the solid boundary. In this paper, the hindered particle movement parallel to the substrate is discussed and measured. The expressions of the drag force and the diffusion coefficient for parallel motion of the sphere relative to the boundary are then expressed as

$$
\begin{gathered}
\mathbf{F}_{\|}=-6 \pi \eta a \mathbf{V} \varepsilon_{\|} \\
D_{\|}=\frac{k_{B} T}{6 \pi \eta a \varepsilon_{\|}}=\varepsilon_{\|}^{-1} D_{0}
\end{gathered}
$$

with $\varepsilon_{\|}$a correction factor to account for the hindered particle movement parallel to the boundary. The expression for this factor in the case of a particle near a single flat boundary does not have a closed analytical form [20]. However, Goldman et al. [21] analyzed the slow viscous motion of a sphere in quiescent viscous fluid and used an asymptotic solution of the Stokes equation to deduce a correction term to account for the hindered particle movement parallel to the boundary, which is expressed as

$$
\varepsilon_{\|}(a, h)=\frac{1}{1-\frac{9}{16}\left(\frac{a}{h}\right)+\frac{1}{8}\left(\frac{a}{h}\right)^{3}-\frac{45}{256}\left(\frac{a}{h}\right)^{4}-\frac{1}{16}\left(\frac{a}{h}\right)^{5}+O\left(\frac{a}{h}\right)^{6}}
$$

with $h(>a)$ the distance from the center of the particle to the surface of the boundary. 


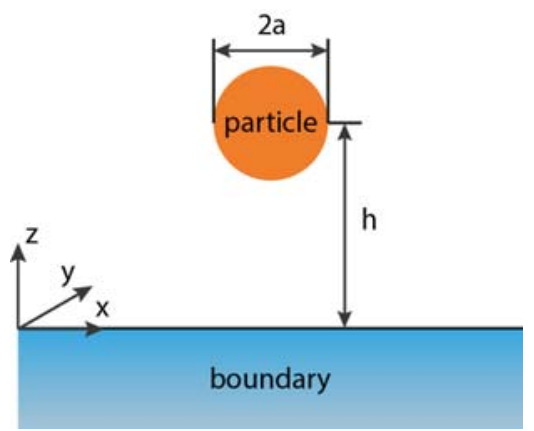

Figure 2. Schematic representation of a Brownian particle in the vicinity of a flat boundary.

The diffusion coefficients of an isolated nanoparticle parallel to a solid boundary can be measured by optical tweezers over a wide range of distances between the particle and the boundary $(190 \mathrm{~nm}<h<10 \mu \mathrm{m})$. By 'isolated', we mean that, aside from the reference particles fixed on the boundary, there is only one particle in an area of $40 \times 40 \mu \mathrm{m}^{2}$. The diffusion coefficient of the nanoparticle in one direction, e.g., in the $x$ direction, $D_{x}$, is defined by

$$
\left\langle\Delta x^{2}(t)\right\rangle=2 D_{x} t
$$

where $\left\langle\Delta x^{2}(t)\right\rangle$ is the mean-squared displacement of the nanoparticle along the $x$-axis over a time $t$.
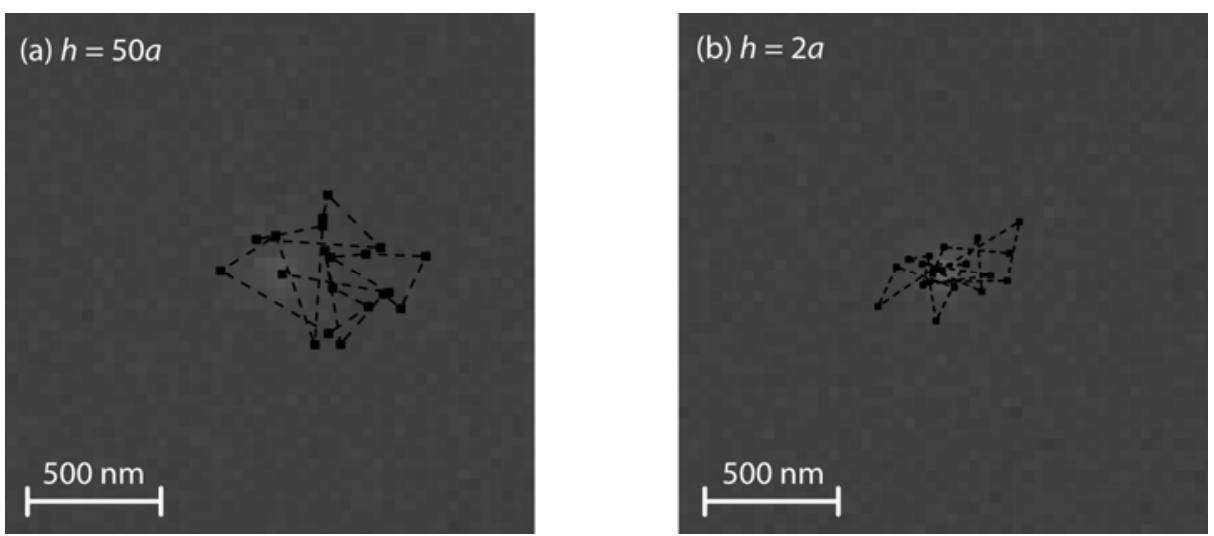

Figure 3. Brownian motion of a $190 \mathrm{~nm}$ particle at the plane where (a) $h=50 a$ and (b) $h=2 a$, respectively. The black dots in each figure show the random positions of the nanoparticle obtained from 20 frames (obtained over a time interval of $0.2 \mathrm{~s}$, $0.01 \mathrm{~s} /$ frame) and the black line segments are the connections between two positions, indicating the absolute displacements of the nanoparicle movement.

The movements of the nanoparticle in different planes parallel to the boundary are directly measured by the QPD, as shown in Figure 3. To derive the diffusion coefficient from Eq. (6) presumes that diffusive transport is observable over the typical observation time of the experiment, which should be possible when this time is smaller than the time it takes for the nanoparticle to travel unhindered in the confinement region of the optical tweezers. Such analysis however requires analysis on larger ensembles of nanoparticles to provide more statistics, so that fluctuations in the stochastic signal get less pronounced. Therefore in our analysis, rather than following a continuous trajectory, our approach is to merely detect discrete and random positions of the nanoparticle within the optical tweezers' confinement region over a timespan of a few seconds, using the fastest image frame rate (100 frame/s) we obtain with our experimental setup. We record the positions of the nanoparticle at this frame rate during an observation time window $t_{\max }$ of 1,2 , and 3 seconds. The temporal displacements of the nanoparticle relative to its starting position, $d(t)$, sampled at $\Delta t=0.01 \mathrm{~s}$, can be obtained by tracing the consecutive positions of the particle 
We then can obtain from this tracking a histogram of the displacement of the nanoparticle relative to its starting point in the confinement region, which should be related to the diffusion coefficient, as detailed below. Indeed, for a single particle, the probability density function for the displacement $d$ is usually defined by a normal law. The width of this Gaussian distribution is characterized by its full width half maximum (FWHM), which is proportional to the standard deviation of the normal distribution. Furthermore, it is known that this standard deviation, which is related to Brownian motion, is proportional to $D^{1 / 2} t^{1 / 2}[22]$.

(a) $t_{\max }=1 \mathrm{~s}, h=1.2 a$

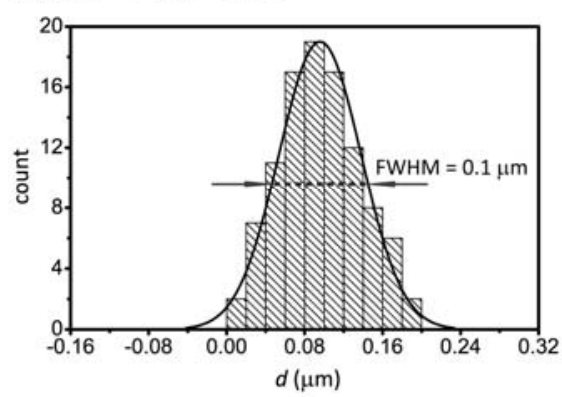

(c) $t_{\max }=3 \mathrm{~s}, h=1.2 a$

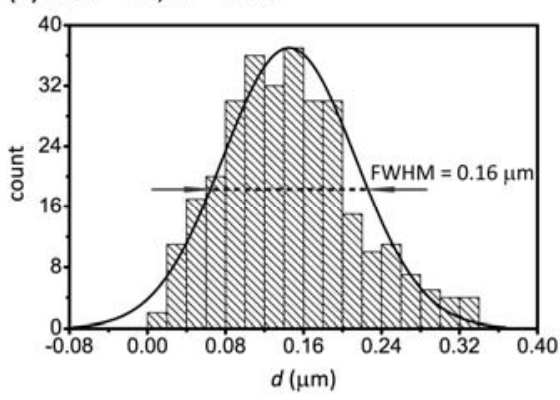

(b) $t_{\max }=2 \mathrm{~s}, h=1.2 a$

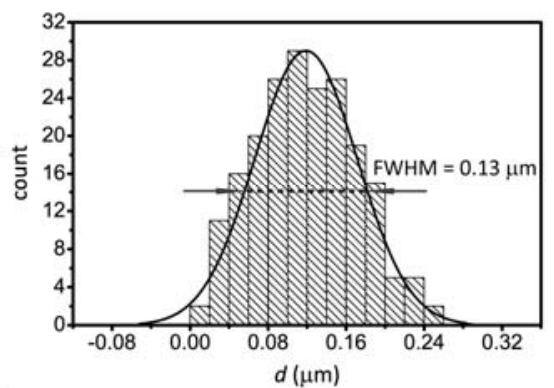

(d)

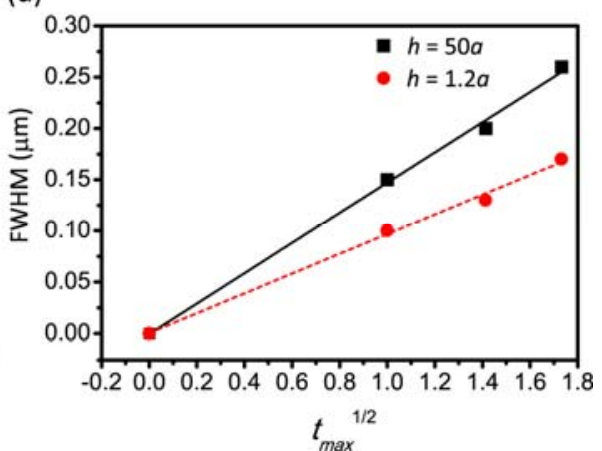

Figure 4. Histograms of the displacements of the nanoparticle relative to its origin position for observation time intervals of (a) $1 \mathrm{~s}$, (b) $2 \mathrm{~s}$, and (c) $3 \mathrm{~s}$, respectively. The results are obtained from a nanoparticle on the position where $h=1.2 a$. The histograms fit with Gaussians, and the FWHM of each Gaussian fit is indicated. (d) The FWHM increases linearly as a function of $t_{\max }{ }^{1 / 2}$. The squares and circles are obtained from a nanoparticle on the plane where $h=50 a$ and $h=1.2 a$, respectively, over the time interval $t_{\max }=1 \mathrm{~s}, 2 \mathrm{~s}$ and $3 \mathrm{~s}$. The solid line is the linear fit of the squares $\left(\mathrm{FWHM}=0.147 \cdot t_{\max }\right.$ $1 / 2)$, and the dashed line is the linear fit of the circles $\left(\mathrm{FWHM}=0.096 \cdot t_{\max }{ }^{1 / 2}\right)$.

The evolution in time of this FWHM can be obtained by considering the scatter plots of the particles positions, and the associated displacements, for different time intervals, for $0 \mathrm{~s}$ to $t_{\max }$ (here, experimentally, the time intervals are $0 \mathrm{~s}$ to $1 \mathrm{~s}$, $0 \mathrm{~s}$ to $2 \mathrm{~s}$ and $0 \mathrm{~s}$ to $3 \mathrm{~s}$ ). The displacements associated to each of these scatter plots can be plotted as histograms, and fit with a Gaussian. The cloud of points associated to the scatter plots is constrained in an envelope, whose size is defined by the diffusion distance, i.e. the standard deviation of the Brownian normal distribution. Also, this standard deviation is related to square root of the sum of the variances of the displacements along the $x$-axis and $y$-axis, thus ensuring the relevance of the analysis. As detailed above, the FWHM of each Gaussian is expected to be characteristic of the diffusion coefficient and the time interval (i.e. proportional to $D^{1 / 2} t^{1 / 2}$ ). As a consequence, the FWHM was obtained from the experimental data (see Figure 4(a)-4(c), for $h=1.2 a$ ), and plotted as a function of $t_{\max }{ }^{1 / 2}$, where $t_{\max }=1 \mathrm{~s}, 2 \mathrm{~s}$ and $3 \mathrm{~s}$. As expected, a clear linear behavior is observed, thus confirming a good agreement with the theory of diffusion and validating our approach. Furthermore, the slope of the linear fit is proportional to $D^{1 / 2}$, thus allowing for a relative quantitation of the variations of $D$ with different experimental parameters (here, different $h$ ).

The diffusive properties of particles are typically obtained by tracking the displacements of a large number of particles, and averaging their trajectories. The obtained data should approximate the probability density function of the diffusion. However, numerous experiments have to be performed to overcome the noise. Indeed, by observing the displacements of a single particle, no clear relation to time could be observed (data not shown). However, with our time interval strategy, the noise due to stochastic displacements can be overcome, as the behavior of the particle is averaged over the time 
interval, thus allowing for a faster analysis of the diffusion of the particle. However, we do not find the absolute value of the diffusion coefficient of Eq. (6) from the slope of the linear fit on Figure 4(d), and therefore, in the following we will always use normalized rather than absolute values when showing the diffusion coefficient, as obtained by the described procedure from our histogram data. This will allow getting relative information on the $z$-dependent behavior of the diffusion process in the $x-y$ plane. In Figure 4, the slope of the fitting for $h=1.2 a$ is much lower than the one obtained for $h=50 a$, thus hinting a decrease in the apparent diffusion coefficient, probably because of restrained diffusion induced by the hindrance of the substrate, preventing displacements in the negative z-direction.

The motion of the nanoparticle parallel to the solid boundary ( $x-y$ plane) is isotropic, as the experimental measurement on the displacements of the nanoparticle along $x$-axis and $y$-axis varies within $5 \%$. The diffusion coefficient obtained from the histogram data of the particle for different planes parallel to the boundary can be obtained, for a fixed time interval, from $0 \mathrm{~s}$ to $t_{\max }$, by comparing the displacement of the particle, i.e.

$$
\text { at } t_{\max }, \frac{D_{\|}(z)}{D_{0}}=\frac{(F W H M)(z)}{(F W H M)(z \rightarrow \infty)}
$$

where $D_{\|}(z)$ is the in-plane diffusion coefficient of the particle at different $z$-axis positions, and $D_{0}$ is the diffusion coefficient when the particle is far away from the boundary. Figure 5 shows the measured values as a function of $h / a$ for an isolated particle confined near a solid boundary, compared with the values calculated from Eq. (5), for the range $1<$ $h / a<50$. For a $190 \mathrm{~nm}$ particle close to the boundary $(h / a \approx 1.2)$, the measured value of $D_{\|}$drops to $\sim 0.4 D_{0}$. When the nanoparticle is about $4 \mu \mathrm{m}$ away from the boundary $(h / a \approx 20)$, it acts as a free Brownian particle.

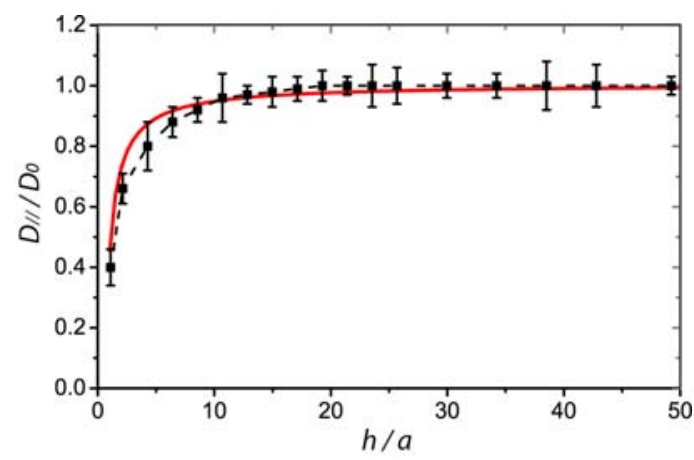

Figure 5. The measured in-plane diffusion coefficient $D \|$ for an isolated $190 \mathrm{~nm}$ particle near a flat solid boundary, normalized by $D_{0}$ (for $t_{\max }=1 \mathrm{~s}$ ). Points represent the average from 3 measurements, error bars the variance, the dashed line is fit for the eye. As comparison, the theoretical correction term shown in Eq. (4) and Eq. (5) is plotted as the solid line.

\section{CONCLUSION}

In this work, the Brownian motion of a single nanoparticle near a solid boundary is characterized by optical tweezers, which provided us with a method to directly determine a diffusion coefficient parallel to the boundary from the histogram data. The method is based on tracking, and then analyzing, the scatter plot of the nanoparticle positions in the confinement field of the optical tweezers. Our results demonstrate that the thus obtained parallel diffusion coefficients are function of the distance from the boundary to the particle and match the theoretical prediction. The study on the dynamics of particles near an interface provides a model system with which we hope to understand in future the phenomenon of complex systems whose surfaces can be modeled as effective boundaries, such as cell membranes.

\section{ACKNOWLEDGEMENT}

Funding of this work was provided by the European Research Council (ERC-2012-AdG-320404) and the Swiss National Science Foundation (200021-152948). 


\section{REFERENCES}

[1] Ashkin, A., Dziedzic, J. M., Bjorkholm, J. E. and Chu, S., "Observation of a single-beam gradient force optical trap for dielectric particles," Opt. Lett. 11, 288-290 (1986).

[2] Ashkin, A., "Optical trapping and manipulation of neutral particles using lasers," Proc. Natl. Acad. Sci.USA 94, 4853-4860 (1997).

[3] Svoboda, K. and Block, S. M., "Biological applications of optical forces," Annu. Rev. Biophys. Biomol. Struct 23, 247-285 (1994).

[4] Pang, Y., Song, H., Kim, J. H., Hou, X. and Cheng, W., "Optical trapping of individual human immunodeficiency viruses in culture fluid reveals heterogeneity with single-molecule resolution," Nature Nanotech. 9, 624-630 (2014).

[5] Block, S. M., "Real engines of creation," Nature 386, 217-219 (1997).

[6] Wang, M. D., "Manipulation of single molecules in biology," Curr. Opin. Biotechnol. 10, 81-86 (1999).

[7] Heller, I., Sitters, G., Broekmans, O. D., Farge, G., Menges, C., Wende, W., Hell, S. W., Peterman, E. J. G. and Wuite, G. J. L., "STED nanoscopy combined with optical tweezers reveals protein dynamics on densely covered DNA," Nat. Methods 10, 910-916 (2013).

[8] Wang, M. D., Yin, H., Landick, R. Gelles, J. and Block, S. M., "Stretching DNA with optical tweezers," Biophys. J. 72, 1335-1346 (1997).

[9] Ando, J., Bautista, C., Smith, N., Fujita, K. and Daria, V. R., "Optical trapping and surgery of living yeast cells using a single laser," Rev. Sci. Instrum. 79, 103705 (2008).

[10]Zhang, H. and Liu, K.-K., "Optical tweezers for single cells," J. R. Soc. Interface 5, 671-690 (2008).

[11]Fällman, E., Schedin, S., Jass, J., Andersson, M., Uhlin, B. E. and Axner, O., "Optical tweezers based force measurement system for quantitating binding interactions: system design and application for the study of bacterial adhesion," Biosens. Bioelectron. 19, 1429-1437 (2004).

[12] Gibson, G. M., Leach, J., Keen, S., Wright, A. J. and Padgett, M. J., "Measuring the accuracy of particle position and force in optical tweezers using high-speed video microscopy," Opt. Express 19, 14561-14570 (2008).

[13] Neuman, K. C. and Nagy, A., "Single-molecule force spectroscopy: optical tweezers, magnetic tweezers and atomic force microscopy," Nat. Methods 5, 491-505 (2008).

[14]Fällen, E. and Axner, O., "Influence of a glass-water interface on the on-axis trapping of micrometer-sized spherical objects by optical tweezers," Appl. Opt. 42, 3915-3926 (2003).

[15] Knöner, G., Parkin, S., Heckenberg, N. R. and Rubinsztein-Dunlop, H., "Characterization of optically driven fluid stress fields with optical tweezers," Phys. Rev. E 72, 031507 (2005).

[16] Wei, M.-T. and Chiou, A., "Three-dimensional tracking of Brownian motion of a particle trapped in optical tweezers with a pair of orthogonal tracking beams and the determination of the associated optical force constants," Opt. Express 13, 5798-5806 (2005).

[17] Banerjee, A. and Kihm, K. D., "Experimental verification of near-wall hindered diffusion for the Brownian motion of nanoparticles using evanescent wave microscopy," Phys. Rev. E 72, 042101 (2005).

[18] Huang P. and Breuer, K. S., "Direct measurement of anisotropic near-wall hindered diffusion using total internal reflection velocimetry," Phys. Rev. E 76, 046307 (2007).

[19] Lin, B., Yu, J. and Rice, S. A., "Direct measurements of constrained Brownian motion of an isolated sphere between two walls," Phys. Rev. E 62, 3909-3919 (2000).

[20] Ha, C., Ou-Yang, H. D. and Pak, H. K., "Study of a colloidal sphere near flat walls using oscillating optical tweezers," Proc. of SPIE 7507, 750702 (2009).

[21] Goldman, A. J., Cox, R. G. and Brenner, H., "Slow viscous motion of a sphere parallel to a plane wall - I Motion through a quiescent fluid," Chem. Eng. Sci. 22, 637-651 (1967).

[22] Saxton, M. J., "Single-particle tracking: the distribution of diffusion coefficients," Biophsics. J. 72, 1744-1753, 1997. 